\title{
Selective Targeting of Bromodomains of the Bromodomain-PHD Fingers Family Impairs Osteoclast Differentiation
}

\section{Julia C. Meier, ${ }^{\dagger}$ Cynthia Tallant, ${ }^{\dagger}$ Oleg Fedorov, ${ }^{\dagger}$ Hanna Witwicka, ${ }^{\ddagger}$ Sung-Yong Hwang, ${ }^{\ddagger}$}

Ruud G. van Stiphout, ${ }^{\|}$Jean-Philippe Lambert, ${ }^{\perp}$ Catherine Rogers, ${ }^{\dagger}$ Clarence Yapp, ${ }^{\dagger}$ Brian S. Gerstenberger, ${ }^{\#}$ Vita Fedele, ${ }^{\dagger}$ Pavel Savitsky, ${ }^{\dagger}$ David Heidenreich, ${ }^{+}$Danette L. Daniels, ${ }^{\nabla}$ Dafydd R. Owen, ${ }^{\#}$ Paul V. Fish, ${ }^{\circ}$ Niall M. Igoe, ${ }^{\circ}$ Elliott D. Bayle, ${ }^{\bigcirc}$ Bernard Haendler, Udo C.T. Oppermann, ${ }^{\text {,I }}$ Francesca Buffa," Paul E. Brennan, ${ }^{\dagger}{ }^{\circ}$ Susanne Müller, ${ }^{\dagger, \S}$ Anne Claude Gingras, ${ }^{\perp, \square}$ Paul R. Odgren, ${ }^{*}$ Mark J. Birnbaum, ${ }^{*}, \boldsymbol{\Lambda}$ and Stefan Knapp ${ }^{*,+, \S,+, \$ \odot}$

${ }^{\dagger}$ Target Discovery Institute and Structural Genomics Consortium, Oxford University, Oxford, United Kingom

${ }^{\ddagger}$ Department of Cell and Developmental Biology, University of Massachusetts Medical School, Worcester, Massachusetts, United States

${ }^{\S}$ Buchmann Institute for Life Sciences (BMLS), Riedberg Campus, 60438 Frankfurt am Main, Germany

"Department of Oncology, Oxford University, Old Road Campus Research Building, Oxford OX3 7DQ, United Kingdom

${ }^{\perp}$ Lunenfeld-Tanenbaum Research Institute, Mount Sinai Hospital, 600 University Avenue, Toronto, Ontario M5G 1X5, Canada

\#Pfizer Worldwide Medicinal Chemistry, 610 Main Street, Cambridge, Massachusetts 02139, United States

${ }^{\nabla}$ Promega Corporation, Madison, Wisconsin, United States

ODepartment of Pharmaceutical \& Biological Chemistry, UCL School of Pharmacy, University College London, 29-39 Brunswick Square, London, WC1N 1AX, United Kingdom

Drug Discovery, Bayer Pharma AG, Müllerstrasse 178, D-13353 Berlin, Germany

"II Botnar Research Centre, Oxford University, Oxford, United Kingdom

${ }^{+}$Goethe-University Frankfurt, Institute of Pharmaceutical Chemistry, Riedberg Campus, 60438 Frankfurt am Main, Germany

$\square$ Department of Molecular Genetics, University of Toronto, Toronto, Ontario M5S 1A8, Canada

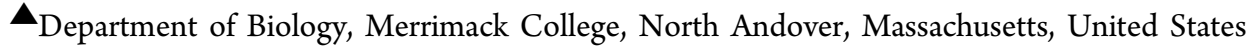

\$German Cancer Network (DKTK), Frankfurt site, 60438 Frankfurt am Main, Germany

Supporting Information
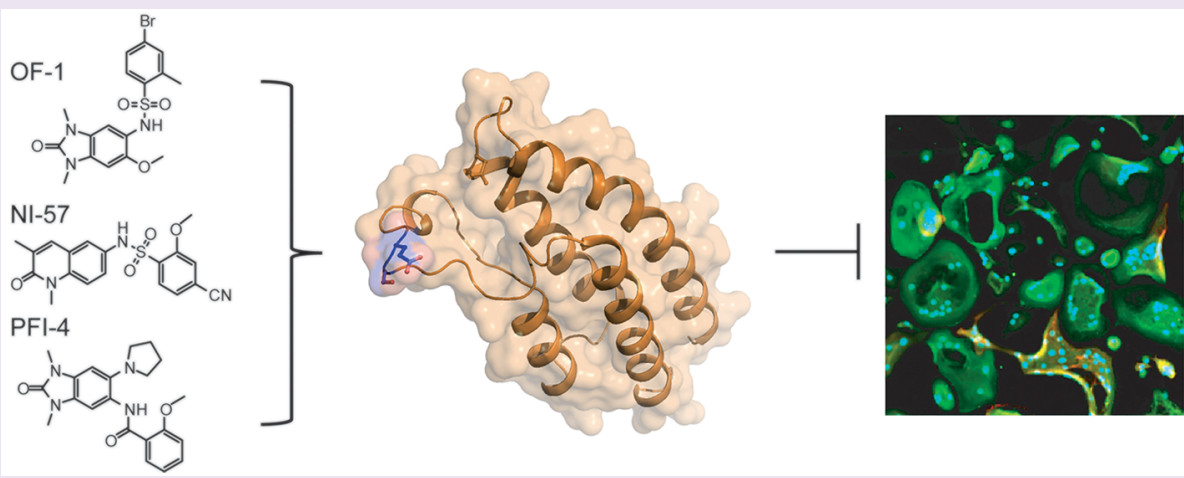

ABSTRACT: Histone acetyltransferases of the MYST family are recruited to chromatin by BRPF scaffolding proteins. We explored functional consequences and the therapeutic potential of inhibitors targeting acetyl-lysine dependent protein interaction domains (bromodomains) present in BRPF1-3 in bone maintenance. We report three potent and selective inhibitors: one (PFI-4) with high selectivity for the BRPF1B isoform and two pan-BRPF bromodomain inhibitors (OF-1, NI-57). The developed inhibitors displaced BRPF bromodomains from chromatin and did not inhibit cell growth and proliferation. Intriguingly, the inhibitors Continued... 
impaired RANKL-induced differentiation of primary murine bone marrow cells and human primary monocytes into bone resorbing osteoclasts by specifically repressing transcriptional programs required for osteoclastogenesis. The data suggest a key role of BRPF in regulating gene expression during osteoclastogenesis, and the excellent druggability of these bromodomains may lead to new treatment strategies for patients suffering from bone loss or osteolytic malignant bone lesions.

A cetylation of histones and other nuclear proteins is a key mechanism regulating gene expression, and aberrant acetylation has been linked to a wide range of diseases. ${ }^{1}$ Histone acetylation is introduced by histone acetyltransferases (HATs) that transfer an acetyl moiety to the $\varepsilon$-amino group of lysine residues. ${ }^{2}$ HATs have usually broad substrate specificity in vitro. In contrast, in vivo, HAT substrate specificity is dramatically enhanced by scaffolding proteins that activate HATs and target them to specific chromatin sites.

Bromodomains are evolutionarily highly conserved proteinprotein interaction modules that act as acetyl-lysine dependent epigenetic reader domains. The human proteome encodes 61 diverse bromodomains that are present in 46 proteins including the HATs CREBBP/EP300 and PCAF/GCN5. ${ }^{3}$ Bromodomains share a conserved fold that comprises a left-handed bundle of four $\alpha$-helices $(\alpha \mathrm{Z}, \alpha \mathrm{A}, \alpha \mathrm{B}, \alpha \mathrm{C})$, linked by loop regions of variable length (ZA and $\mathrm{BC}$ loops), which line the KAc binding site and determine binding specificity. Due to its excellent druggability, the bromodomain acetyl-lysine binding pocket has emerged as an attractive site for the development of protein interaction inhibitors. ${ }^{4}$ Highly potent and selective inhibitors have been developed for BET (BRD2, BRD3, BRD4, BRDT) bromodomains, ${ }^{5-8}$ and a number of BET inhibitors have now entered clinical testing. ${ }^{9}$ While to date most efforts have focused on BET inhibitor development, recent publications have demonstrated that non-BET bromodomains can also be selectively targeted. ${ }^{10-18}$ A first inhibitor specific for the BRPF1B bromodomain has been recently disclosed, ${ }^{19}$ and inhibitors that showed dual activity for the bromodomains present in BRPF 1 and TIF $1 \alpha$ have been developed by our laboratory and others. ${ }^{20,21}$ However, phenotypic consequences of inhibiting protein interactions mediated by BRPF bromodomains have not been reported so far.

The MYST ( $\underline{M O Z}, \underline{Y} b f 2 / S a s 3, \underline{S} A S 2$ and Tip60) family of lysine acetyl-transferases form signaling complexes with heterotetrameric core structures comprising a MYST family member, the ING tumor suppressor, hEAF6 (an EPC (enhancer of polycomb)associated protein), and a central scaffolding protein of the BRPF (Bromodomain-PHD fingers) family. In higher eukaryotes, the BRPF family contains three members (BRPF1, BRPF2 (also called BRD1), and BRPF3) with conserved domain architecture of two N-terminal PHD domains linked by a $\mathrm{Zn}^{2+}$ knuckle (PZP [PHD-Zn knuckle-PHD] domain), a bromodomain, and a C-terminal PWWP domain. ${ }^{22,23}$ The BRPF PHD domains target unmethylated histone $\mathrm{H} 3{ }^{24}$ The PWWP domains recognize the H3K36me3 mark, and the bromodomains preferentially interact with $\mathrm{H} 2 \mathrm{AK} 5 \mathrm{ac}, \mathrm{H} 4 \mathrm{~K} 12 \mathrm{ac}$, and $\mathrm{H} 3 \mathrm{~K} 14 \mathrm{ac}{ }^{25}$ BRPF1 associates with MOZ/MORF, assembling a signaling complex that plays a role in maintaining anterior HOX gene expression during development. ${ }^{26} \mathrm{MOZ}$ is frequently translocated in acute myeloid leukemia (AML), and it is required for hematopoietic stem cell maintenance. ${ }^{27}$ Biochemical studies have shown that BRPF1 still interacts with and enhances the transcriptional potential of the leukemic MOZ-TIF2 fusion protein. ${ }^{28}$ BRPF2 preferentially associates with $\mathrm{HBO} 1$, assembling a chromatin complex required for global acetylation of H3K14ac. HBO1/BRPF2 plays a key role in the regulation of erythropoiesis. In mice, deletion of the BRPF2 gene results in severe anemia due to impaired fetal liver erythropoiesis. ${ }^{29}$ In addition, polymorphism in BRPF2 has been recently linked to bipolar disorder and schizophrenia. ${ }^{30}$ The related JADE scaffolding proteins that lack the $\mathrm{C}$-terminal bromo and PWWP domains can replace BRPF2 in the HBO1 complex. Intriguingly, this exchange in scaffolding protein determines which histone tail is acetylated: the JADE complex directs $\mathrm{HBO} 1$ toward the $\mathrm{H} 4$ tail, whereas BRPF2 confers high selectivity for $\mathrm{H} 3$. $^{24}$ The central role of reader domains in MYST complexes and the key role of these complexes in hematopoiesis prompted us to study the consequences of pharmacological targeting of BRPF readers of the bromodomain family in monocyte differentiation, with osteoclasts representing a particularly well-studied and clinically relevant monocyte-derived lineage. Here, we report that selective pharmacological inhibition of BRPF bromodomains but not of the BRPF1B bromodomain alone strongly impaired RANKL-induced differentiation of murine and human primary monocytes into bone resorbing osteoclasts. Genomewide mRNA expression analysis showed that panBRPF bromodomain inhibition suppressed transcriptional programs required for osteoclastogenesis, establishing a role of BRPF family members in bone degradation. The data indicate that interactions mediated by BRPF bromodomains play a central role in bone maintenance and may be attractive targets for the development of drugs preventing osteoporosis and metastasis or cancer induced osteolysis.

\section{RESULTS AND DISCUSSION}

The human BRPF family (BRPF1, BRPF2, and BRPF3) shares a conserved domain architecture $\mathrm{e}^{22,23}$ and a high degree of sequence homology within their bromodomains (Figure 1A,B). Interestingly, we found that alternative splicing generates two BRPF1 isoforms ( $\mathrm{A}$ and $\mathrm{B}$ ). The longer BRPF1A harbors a six-residue insert in the ZA-loop that prevented binding to histone peptides as well as inhibitors (Figure 1C). Thus, it seems that the BRPF1 bromodomain is regulated by alternative splicing, which generates a dominant negative isoform. Unfortunately, we were unable to crystallize the $\mathrm{A}$ isoform, but the location of the inset within the ZA loop region and the inability of this isoform to bind inhibitors suggested that BRPF1A specific insertion blocks access to the acetyl-lysine binding site (Figure 1D).

To enable identification of BRPF bromodomain inhibitors, we developed an ALPHAscreen (amplified luminescent proximity homogeneous assay) assay using a tetra-acetylated histone 4 peptide (H4K5acK8acK12acK16ac) and his ${ }_{6}$-tagged recombinant BRPF bromodomains. Screening of an in-house bromodomain targeted library and fragment sets resulted in the identification of a number of inhibitors that carried either the benzoimidazolone or the dimethylquinolinone core structure. Purchasing and optimization by synthetic medicinal chemistry efforts led to the development of the potent benzoimidazolone-based inhibitors OF-1, PFI-4, and the dimethylquinolinone NI-57 (Figure 2A). A detailed discussion of the SAR (structure-activity relationship) will be reported elsewhere. ${ }^{31,32}$ Using dose response ALPHAscreen assays, the developed chemical probes showed potencies for BRPF1B $\left(\mathrm{IC}_{50}\right)$ of $270 \mathrm{nM}, 172 \mathrm{nM}$, and $114 \mathrm{nM}$ for OF-1, PFI-4, and NI-57, respectively. These data correlated well with temperature shift and isothermal titration calorimetry data (ITC), but ALPHAscreen underestimated somewhat the affinity of 


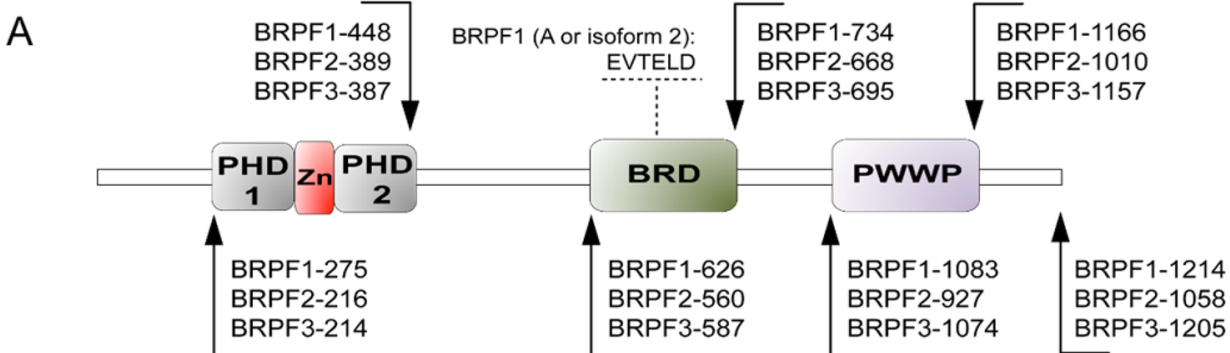

B

hBRPF1B
hBRPF1A
hBRPF2
hBRPF3

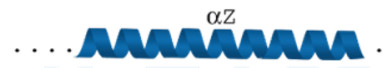$$
\text { ZA-loop }
$$$$
\text { BRPF3-1205 }
$$
MQLTPFLILLRKTLEQLQEKDTGNIFSEPVPLS - . - - - EVPDYILDHIKKPMDFFTMKQNLEAY MQLTPFL I LLRKTLEQLQEKDTGN I F SEPVP L SEVTELDEVPDYLDH I KKPMDF FTMKQNLEAY LRLTPLTVLLRSVLDQLQDKDPAR I FAQPVS L K - - - - EVPDYLDHI KHPMDFATMRKRLEAQ LELMP FNVLLRTTLDLLQEKDPAHI FAEPVNLS - - - - EVPDYLEF I SKPMDFSTMRRKLESH

$$
\mathrm{AB}-100 \mathrm{p} \sim \mathrm{BC} \mathrm{B}^{\mathrm{B}-100 \mathrm{p}}
$$

hBRPF1B RYLNFDDFEEDFNLIVSNCLKYNAKDTIFYRAAVRLREQGGAVLRQARRQAEKMGID hBRPF1A RYLNFDDFEEDFNLIVSNCLKYNAKDTI FYYRAAVRLREQGGAVLRQARRQAE KMGID hBRPF2 GYKNLHEFEEDFDLIIDNCMKYNARDTVFYRAAVRLRDQGGVVLRQARREVDSIGLE hBRPF3 LYRTLEEFEEDFNLIVTNCMKYNAKDTIFHRAAVRLRDLGGAILRHARRQAENIGYD
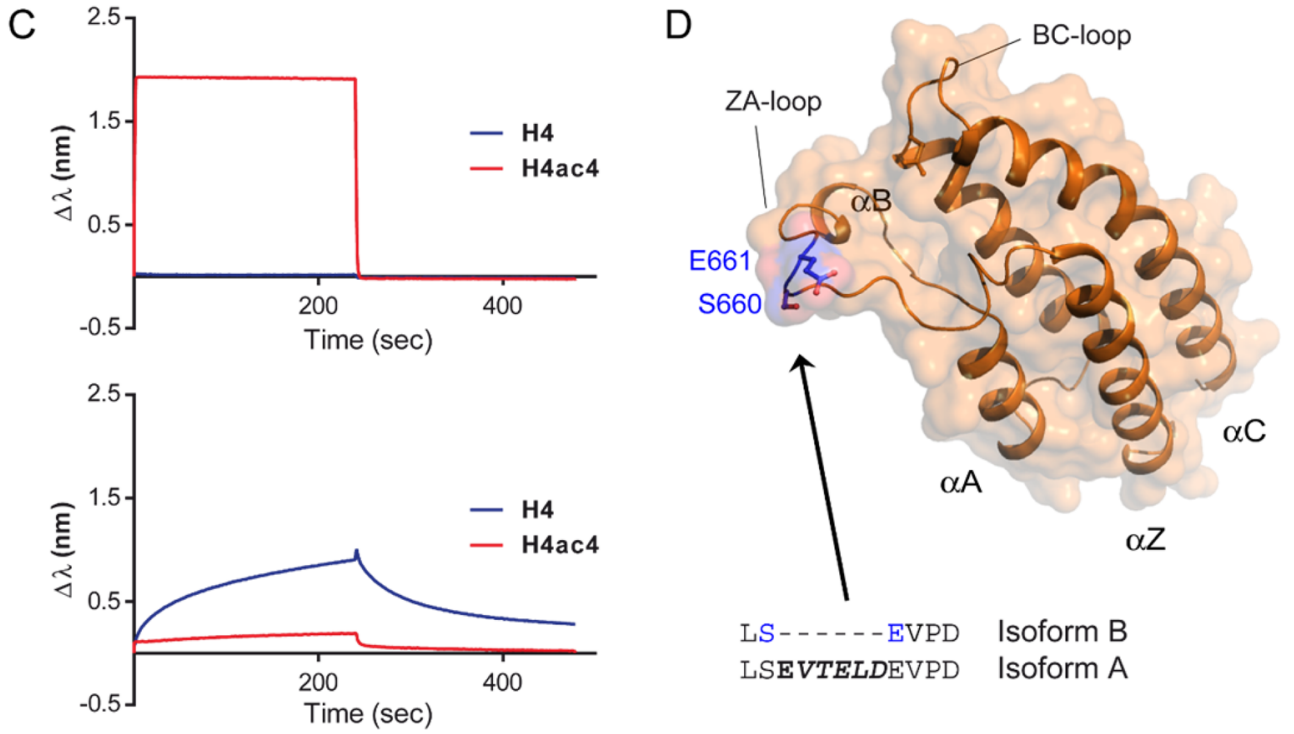

Figure 1. BRPF bromodomain family and its inhibitors. (A) Domain organization of human BRPF proteins. Two splice isoforms of BRPF1B are expressed (A and B) that differ in the ZA loop of the bromodomain. In BRPF1A (or isoform 2), six residues EVTELD (661-666) are inserted into the ZA loop. Annotated domains are the PHD (plant homeo-domain) connected by a zinc finger, the bromodomain (BRD), and the PWWP domain (harboring the PWWP motif). (B) Sequence alignment of human BRPF bromodomains. The main secondary structural elements are highlighted. (C) BLI (BioLayer Interferometry) data measured on the two splice isoforms of BRPF1A and BRPF1B. Shown are the raw data traces for acetylated as well as nonacetylated peptide. (D) Location of the isoform BRPF1A specific insertion depicted on the structure of BRPF1B.

PFI-4 for the BRPF1B isoform, which had a $K_{\mathrm{D}}$ of $13 \pm 1 \mathrm{nM}$ using ITC (Supporting Information Tables $1-3$ ).

Next, we used temperature shift assays $\left(\Delta T_{\mathrm{m}}\right)$ to evaluate the family wide selectivity of the three developed chemical probes using a comprehensive panel of 49 diverse bromodomains. OF-1 showed significant $\Delta T_{\mathrm{m}}$ shifts within the BRPF family identifying this inhibitor as a pan-BRPF inhibitor, but also weak interactions $\left(2.1{ }^{\circ} \mathrm{C}\right)$ were observed for BRD4(1). $\Delta T_{\mathrm{m}}$ shifts of $1^{\circ}$ were observed for other bromodomains such as other BET family members, TRIM24 (TIF $1 \alpha$ ), and BRD9 (Figure 2B). However, such small $\Delta T_{\mathrm{m}}$ shifts often represent false positive hits or very weak interactions. Indeed, ALPHAscreen did not reveal strong interactions of OF-1 with BRD4 and TRIM24 (TIF1 $\alpha)$, with $\mathrm{IC}_{50}$ values larger than $10 \mu \mathrm{M}$ (Supporting Information Table 2). ITC determined a $K_{\mathrm{D}}$ of $3.9 \pm 0.3 \mu \mathrm{M}$ for the first bromodomain of BRD4(1), thus 39-fold selectivity when compared to the
BRPF1B isoform (Figure 2C). Using ITC, we determined $K_{\mathrm{D}}$ values of $0.5 \pm 0.06 \mu \mathrm{M}$ and $2.4 \pm 0.2 \mu \mathrm{M}$ for BRPF2 and BRPF3, respectively. We did not identify any significant interaction outside subfamily IV for NI-57, which showed only weak $\Delta T_{\mathrm{m}}$ shifts $\left(\sim 1{ }^{\circ} \mathrm{C}\right)$ for the bromodomain present in BRD9, CREBBP, and EP300, suggesting excellent selectivity for the BRPF family. ITC revealed a $K_{\mathrm{D}}$ of $0.031 \pm 0.002 \mu \mathrm{M}$ for BRPF1B (Supporting Information Table 3), in agreement with ALPHAscreen data $\left(\mathrm{IC}_{50}: 0.114 \pm 0.061 \mu \mathrm{M}\right)$. Finally, PFI-4 was highly selective for the BRPF1B isoform. $\Delta T_{\mathrm{m}}$ screening against the bromodomain family detected only a weak temperature shift for the CECR2 bromodomain. ITC confirmed isoform selectivity for BRPF1B $\left(K_{\mathrm{D}}: 0.013 \pm 0.001 \mu \mathrm{M}\right)$, whereas BRPF2 interacted with this chemical probe with a $K_{\mathrm{D}}$ of $0.775 \pm 0.09 \mu \mathrm{M}$ (60-fold selectivity) and CECR2, the only detected off-target outside family IV with only $2.35 \pm 0.52 \mu \mathrm{M}$ affinity, thus showing 180 -fold selectivity. 


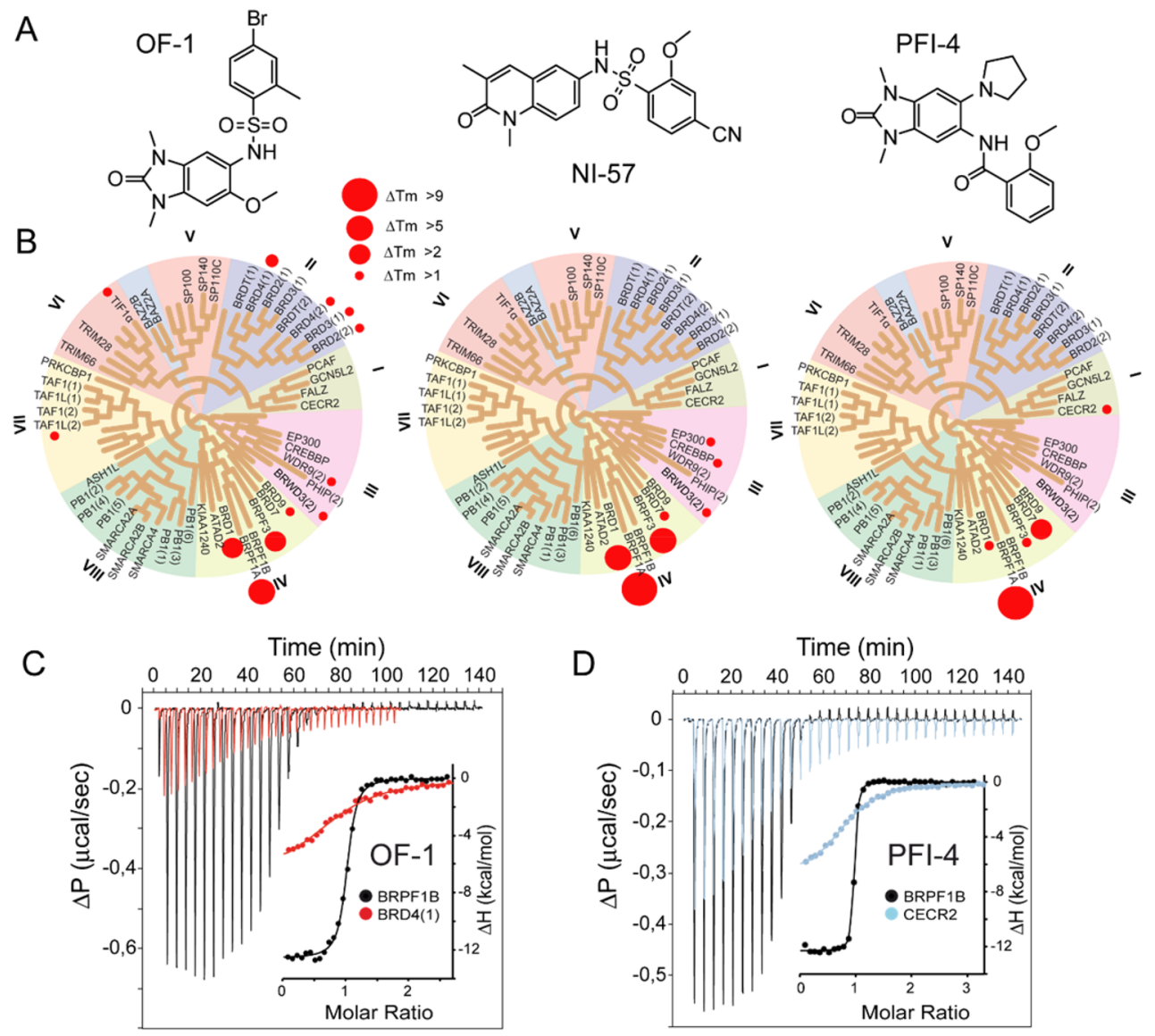

Figure 2. Selectivity and potency of the three BRPF chemical probes. (A) Chemical structure of OF-1, NI-57, and PFI-4. (B) Selectivity screening data of OF-1, NI-57, and PFI-4 using temperature shift assays $\left(\Delta T_{\mathrm{m}}\right)$. The temperature shifts (listed in Supporting Information Table 1) were mapped onto the phylogenetic tree using red spheres with radii corresponding to $\Delta T_{\mathrm{m}}$ as indicated in the figure. (C) ITC measurements of the OF-1 with BRPF1B and its main off-target BRD4 domain 1 (shown in red). Raw binding heats are shown for each injection. The insert depicts normalized binding heats and a nonlinear least-squares fit to a single binding site model. (D) ITC measurements of PFI-4 with BRPF1B and its main off-target CECR2. See also, Supporting Information Tables $1-4$.

BRD7 was detected in $T_{\mathrm{m}}$ assays, but this interaction was not confirmed in alternative assays (DiscoverX bromoscan) probably due to the low intrinsic stability of the protein leading often to misleading DSF results (Figure 2D). It is interesting to note that in agreement with our peptide binding data, none of the inhibitors interacted with the BRPF1A isoform in temperature shift assays, consistent with this splicing isoform acting as a bromodomain inactivating variant with an inaccessible acetyl-lysine binding site. Screening of the developed probes against a diverse panel of potential off-targets (kinases, GPCRs) revealed no significant off-targets outside the bromodomain family (Supporting Information Table 4). In conclusion, medicinal chemistry and in vitro screening efforts led to the development of three potent chemical tools with good selectivity for the BRPF family as well as one highly isoform-selective chemical probe. Thus, this set of three chemical probes allows independent evaluation of phenotypic consequences of BRPF bromodomain inhibition as well as BRPF1B specific activities in cellular systems.

Following the analysis of inhibitor potency and selectivity in vitro, we set out to demonstrate cellular "on-target" activity of the three probe molecules. Since the developed inhibitors are anticipated to block chromatin association of BRPF, it is expected that the inhibitors would weaken the interactions of BRPF with histones and strongly inhibit the recruitment of isolated BRPF bromodomains to histones. To assess this, we developed a BRPF1A/B-Histone H3.3-nanoBRET (nano bioluminescence resonance energy transfer) assay, which measures the energy transfer from one NanoLuciferase coupled protein (BRPF1, donor) to the interacting HaloTag-protein labeled with a NanoBRET 618 fluorophore (histone H3.3 acceptor). Indeed, we observed dose-dependent displacement of BRPF1B but not of the BRPF1A isoform from histone H3.3 (Figure 3, Supporting Information Figure 1). Estimated $\mathrm{IC}_{50}$ values were $0.07 \pm 0.0034$ and $0.24 \pm 0.039 \mu \mathrm{M}$ for NI-57 and PFI-4, respectively. We verified these data using FRAP (fluorescence recovery after photobleaching) assays. ${ }^{33}$ As expected from our selectivity screening data, PFI-4 led only to the dissociation of the bromodomain of BRPF1b, but not any of the full-length family members from histone H3.3 (Supporting Information Figure 1B). NI-57 displaced a GFP fusion construct where the bromodomain was triplicated as well as full-length GFP-BRPF2, but not inactivating bromodomain mutants from chromatin, which was indicated by significant reduction of recovery times in the presence of the inhibitor. Acetylation dependence of the interaction was demonstrated by adding the pan-HDAC inhibitor suberoylanilide hydroxamic acid (SAHA), which leads to a global increase in histone acetylation and therefore to stronger association of the BRPF bromodomains with histones (Figure 3B-D). Similar results were observed for OF-1 and PFI-4 (Supporting Information Figure 1). Thus, the nanoBRET and FRAP 

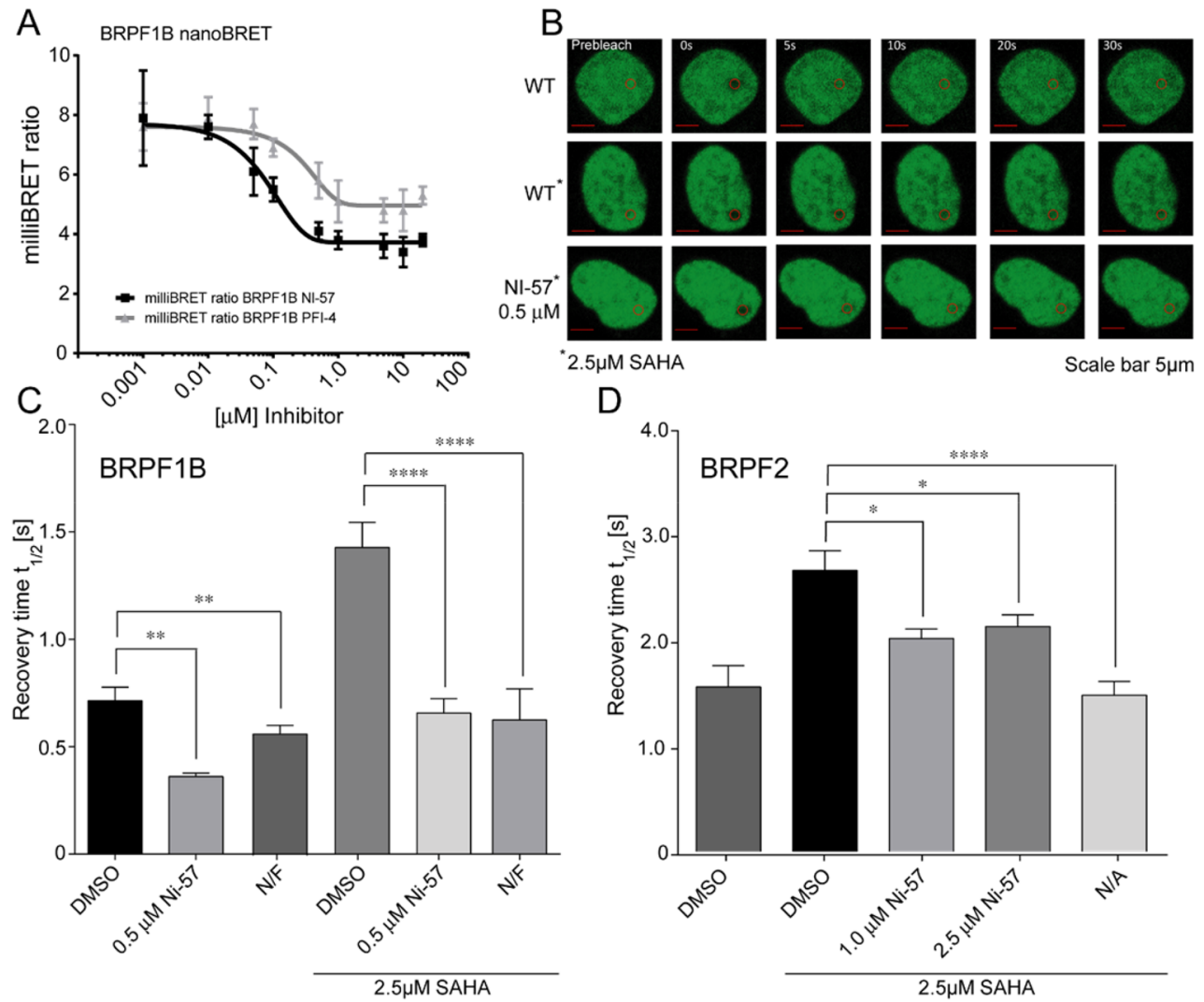

D

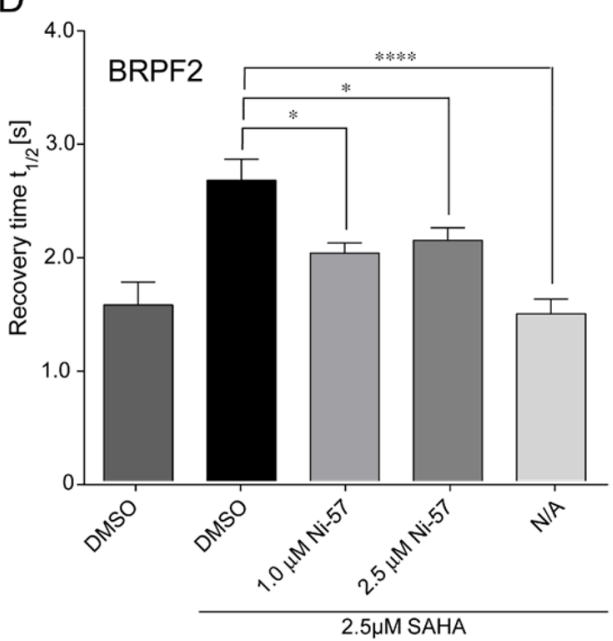

Figure 3. Inhibition of BRPF bromodomains in the nucleus. (A) Dose-dependent inhibition of the BRPF1B and histone H3.3 protein interaction with NI-57 and PFI-4 measured by NanoBRET assay. (B) Representative confocal images of nuclei from U2OS cells transfected with plasmids encoding triple bromodomains of BRPF1B treated either with or without SAHA $\left(^{*}\right)$ and the panBRPF Inhibitor NI-57. The bleached area is indicated by a red circle. (C) Half-times of fluorescence recovery $\left(t_{1 / 2}\right)$ after photo bleaching measured for the BRPF1B triple bromodomain construct. (D) Half-times of fluorescence recovery $\left(t_{1 / 2}\right)$ after photo bleaching measured for full-length BRPF2 (WT) after treatment with NI-57 at different concentrations with or without SAHA. Bars in panel C and D represent the mean $t_{1 / 2}$ calculated from at least 10 individual recovery curves, and error bars depict the standard error of the mean. ${ }^{*} p<0.05, * * p<0.01, * * * p<0.001, * * * * p<0.0001$ significant difference from wild type with or without SAHA ( ${ }^{\ddagger} 2.5 \mu \mathrm{M}$; $\mathrm{n}$-way ANOVA and Dunnett's posthoc-test). See also, Supporting Information Figure 1.

experiments demonstrated that the developed inhibitors strongly inhibit BRPF1 and BRPF2 but not BRPF1A bromodomains in the nucleus.

Structural models of monoacetylated histone peptides $\mathrm{H} 2 \mathrm{AK} 5 \mathrm{ac}$ and $\mathrm{H} 4 \mathrm{~K} 12 \mathrm{ac}$ have been published recently, revealing a canonical bromodomain acetyl-lysine interaction. ${ }^{25}$ However, we wanted to confirm the binding mode of peptides that we used in screening assays and for which we detected the tightest association with BRPF1B. In particular, we were interested in the consequences of the presence of multiple acetylation sites on histone recognition as well as the recognition of the histone H3 mark H3K14ac. We therefore cocrystallized BRPF1B with peptides harboring the $\mathrm{H} 3 \mathrm{~K} 14 \mathrm{ac}$ and $\mathrm{H} 4 \mathrm{~K} 5 \mathrm{acK} 8 \mathrm{ac}$ mark. The $\mathrm{H} 3 \mathrm{~K} 14 \mathrm{ac}$ complex revealed the canonical interaction of the acetyl-lysine with the BRPF1B bromodomain comprising the conserved hydrogen bond with N708 as well as the watermediated hydrogen bond with Y665 and additional hydrogen bonds formed by the H3R17 side chain and the backbone carbonyl of the G650 (Supporting Information Figure 2A-C). It is interesting to note that in the H3K14ac complex the peptide reversed its orientation when compared to complexes of the same mark with the bromodomain of BAZ2B. ${ }^{34}$ Cocrystallization of the diacetylated peptide $\mathrm{H} 4 \mathrm{~K} 5 \mathrm{acK} 8 \mathrm{ac}$ revealed that in contrast to cocrystal structures with $\mathrm{BRD} 4^{35}$ only $\mathrm{H} 4 \mathrm{~K} 5 \mathrm{ac}$ interacted with the acetyl-lysine binding site, probably due to steric constraints of the bulky residue F714 preventing simultaneous interaction of two acetylated lysines in BRPF1B (Figure 4A). In the cocrystal structure, the H4K8ac side-chain was oriented toward the surface but in close proximity to an area of strongly positive electrostatic potential. It is therefore likely that neutralization of the positive charge of the lysine by acetylation contributes favorably to the interaction with this bromodomain.

We cocrystallized OF-1 as well as PFI-4 to confirm the acetyllysine mimetic binding mode suggested by our peptide displacement screening assays and to elucidate the structural mechanisms of the observed selectivity. As expected, the benzimidazolone acted as an acetyl-lysine mimetic moiety forming in the BRPF1B complex the canonical hydrogen bond between the conserved asparagine (N708) and the characteristic water-mediated hydrogen bond with Y665 (Figure 4B,D). The inhibitor was further stabilized by a number of hydrophobic interactions with lipophilic groups located at the rim of the Kac binding site. The sulphonamide linker caused a $90^{\circ}$ bend, positioning the bromo-methylphenyl ring on top of F714, allowing an aromatic edge-face stacking interaction and hydrophobic contacts with I713. Comparison with the BRPF2-OF1 complex showed conservation of the binding mode, but the bromo-methylphenyl ring assumed a position that is turned away from F714 due to rotation of the phenyl ring (Supporting Information Figure 4D). Sequence conservation in the acetyl-lysine binding pocket of BRPF1B and BRPF2 is high, but the BC-loop residue $\mathrm{I} 713$ and the central ZA-loop residue $\mathrm{P} 658$ positions are substituted by 

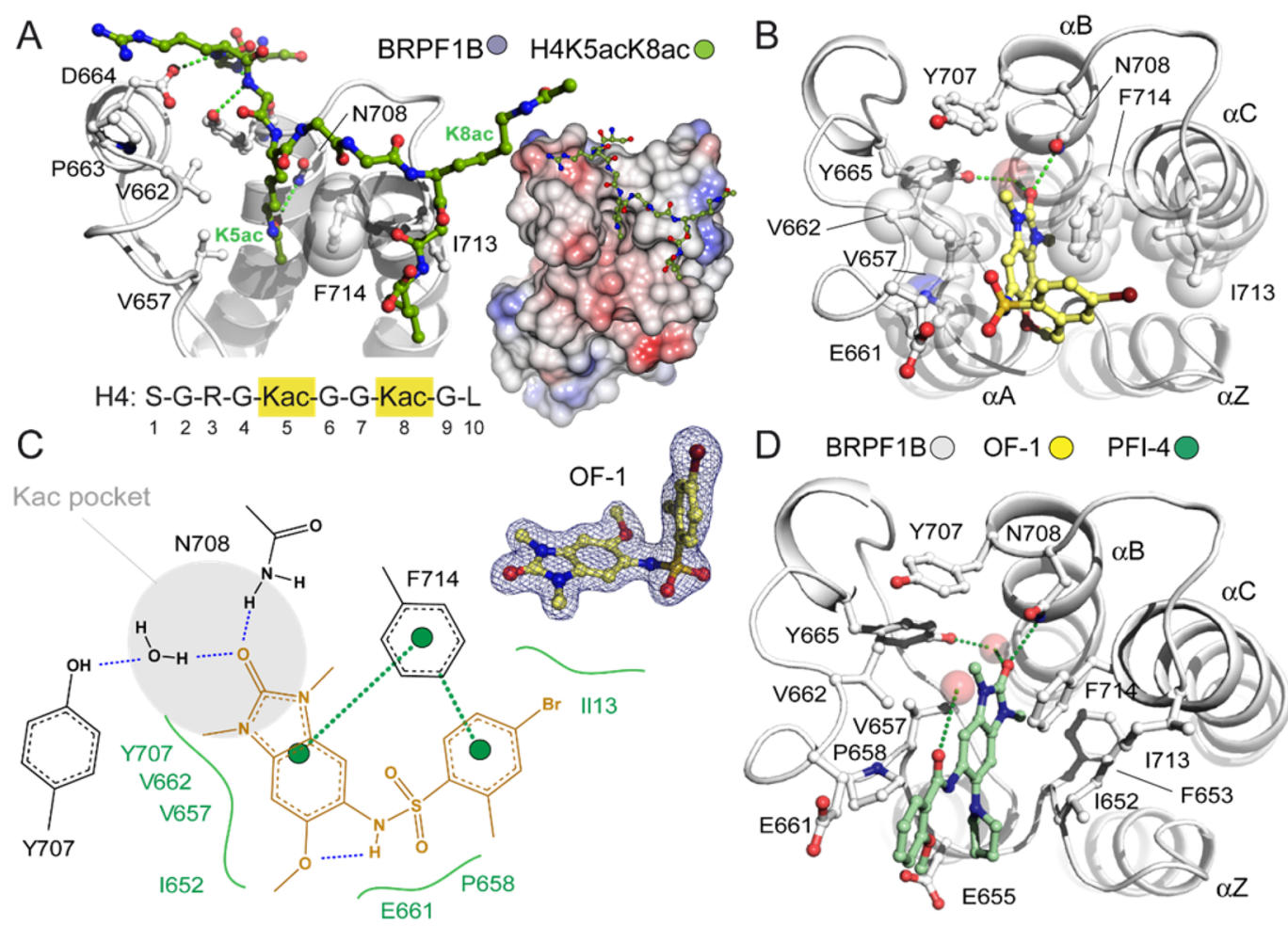

Figure 4. Substrate recognition and inhibitor binding modes. (A) Details of the interaction of H4K5acK8ac with BRPF1B. The inset on the right shows a surface representation indicating the electrostatic potential ranging from $+1.5 \mathrm{~V}$ (blue) to $-1.5 \mathrm{~V}$ (red). (B) Details of the interaction of OF-1 with the BRPF1B bromodomain. OF-1 is shown in ball and stick representation. Hydrogen bonds are shown as dotted lines. (C) 2D projection showing the interactions of OF-1 with the BRPF1B acetyl-lysine binding site. Blue dashed lines represent hydrogen bonds; green solid lines, hydrophobic interactions; and green dashed lines, $\pi-\pi$ stacking and edge-to-face aromatic interactions. The panel on the top right shows a $2 \mathrm{~F}_{\mathrm{o}}-\mathrm{F}_{\mathrm{c}}$ electron density map contoured at $1.2 \sigma$ around the inhibitor at $1.65 \AA$. (D) Details of the interaction of the BRPF1B bromodomain with PFI-4. See also Supporting Information Figure 2 and Supporting Information Table 5.

V647 and S592 in BRPF2. This creates a larger and shallower binding pocket, resulting in repositioning of OF-1. ITC data suggested that higher affinities of the developed inhibitors for the BRPF1B isoform are mainly due to a more favorable binding enthalpy (Supporting Information Table 3). It is therefore likely that the less efficient stacking of the bromo-methylphenyl ring with F714 as well as the lack of interaction of the halogen atom with the adjacent binding pocket formed by I713 are the main reasons for the 5-fold weaker potency of OF-1 for BRPF2. Comparison with the BRPF1B-PFI-4 complex revealed that the amide linkage with the benzoimidazolones ring system does not allow orientation of the methoxybenzamide toward F714 (Figure 4D, Supporting Information Figure 2E,F). The orientation of the PFI-4 methoxybenzamide ring is additionally stabilized by the presence of an intramolecular hydrogen bond, resulting in an orientation toward the ZA loop. Together with the 6-pyrrolidine substituent, the inhibitor shows remarkable shape complementarity with the BRPF1B acetyl-lysine binding site, explaining the high potency for this target. Crystallographic data and refinement statistics are summarized in Supporting Information Table 5.

To understand the complexity of the BRPF signaling network, we used proximity-dependent protein biotinylation (BioID) to identify physiologically relevant BRPF protein interactions in living cells using HEK293 as a model. ${ }^{36}$ The experiments revealed the canonical BRPF core complex structure, comprising MYST acetyltransferases as well as MYST/Esa1-associated factor 6, which was found preferentially associated with BRPF3, and the general complex components ING4/5, in addition to other associated proteins that were often isoform specific (Figure 5A). ING4/5 associated with all BRPF family members, but it is interesting to note that MYST family members preferentially associate with specific BRPF isoforms. HBO1 (KAT7) was reported to preferentially interact with BRPF2, but significant association with BRPF3 was also detected. KAT6A (MOZ) preferentially associated with BRPF1B, in agreement with published data, ${ }^{26}$ but MORF (KAT6B) also showed significant interaction with a short isoform of BRPF2. Also interesting was the presence of Ser/Thr phosphatases (PPP1CC) and their regulators as well as the members of the casein kinase family, which were found in BRPF1B complexes, suggesting crosstalk with phosphorylation dependent signaling events (Supporting Information Figure 3).

Epigenetic mechanisms play an important role in patterning and differentiation processes, and BRPF and its associated HATs have been particularly associated with differentiation of hematopoietic cells. Given our interest in bone biology, and the hematopoietic origin of the osteoclast, we investigated the potential role of the targeted bromodomains in osteoclastogenesis in mouse and human cells. Bone marrow mononuclear cells were isolated from wild-type mice, cultivated for 5 days in the presence of CSF-1, and replated in the presence of RANKL for differentiation into osteoclasts, ${ }^{28}$ either with DMSO as a control or with varying doses of the BRPF inhibitors. OF-1 and NI-57 both caused significant reductions in the number of multinucleated tartrate-resistant acid phosphatase (TRAP) positive cells (Figure 5B, Supporting Information Figure 4). To understand the molecular basis for this observation, we investigated the expression of several osteoclast marker genes using qPCR. As expected, we found significant reductions of 


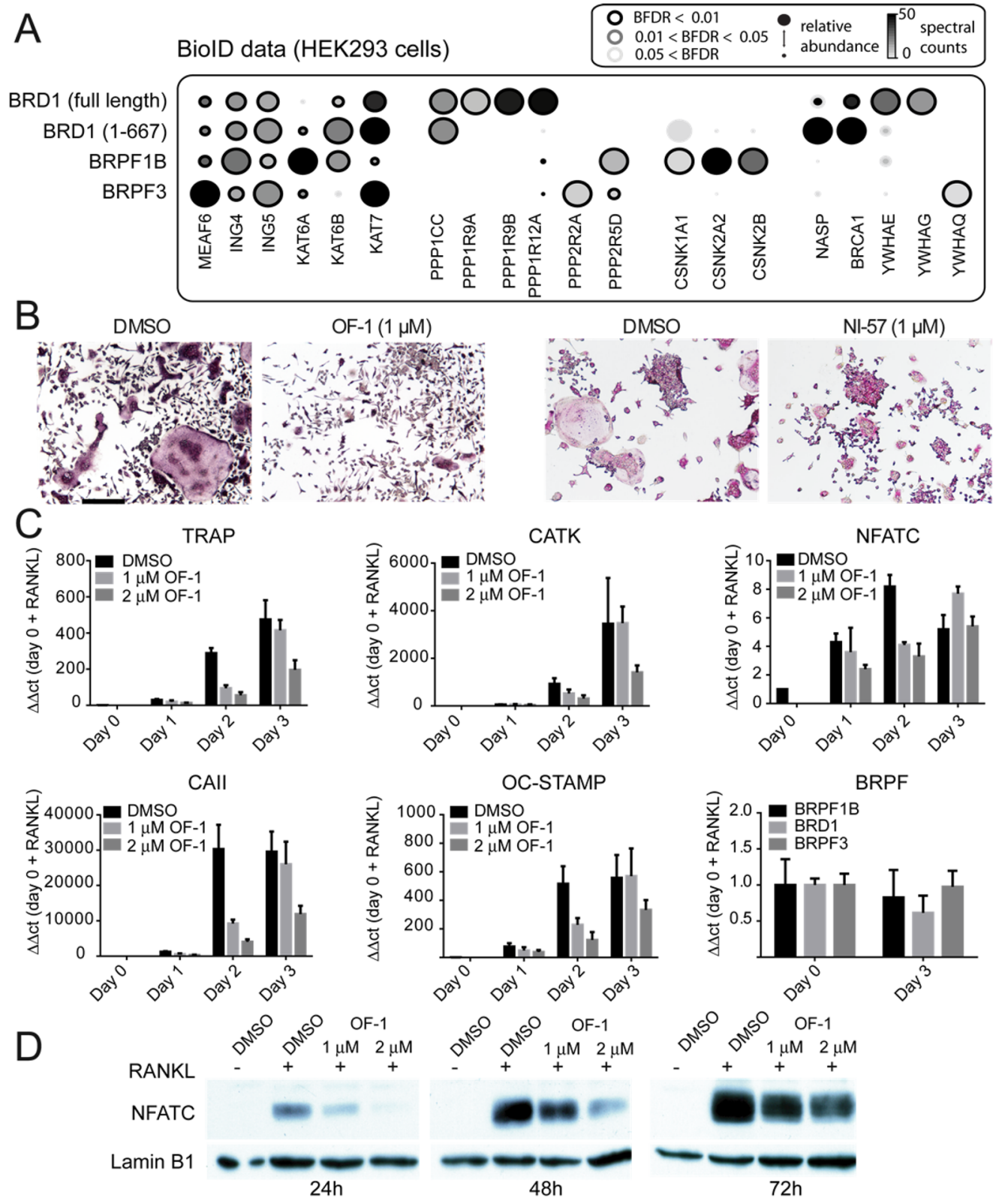

Figure 5. BRPF1B binding partners and effect of BRPF bromodomain inhibition on gene transcription during mouse osteoclastogenesis. (A) BioID of full-length and truncated (1-867) BRPF2, BRPF1B, and BRPF3 in HEK293 cells. The color of the circle represents the absolute spectral counts observed for the prey proteins. The circle size indicates the quantitative enrichment of a prey across all baits tested while the estimated FDR for the interaction is shown as the color of the circle edge. (B) Inhibition of murine osteoclast differentiation by panBRPF bromodomain inhibition. TRAP stain of primary murine bone marrow cells (BMCs) differentiated into osteoclasts for 3 days with $1 \mathrm{ng} / \mathrm{mL}$ RANKL plus treatment with DMSO or the panBRPF bromodomain inhibitor OF-1. Bar scale indicates $200 \mu \mathrm{m}$. (C) RNA expression of osteoclast markers and BRPF $1,-2$, and -3 in murine BMCs during differentiation with $10 \mathrm{ng} / \mathrm{mL}$ RANKL and treatment with $1 \mu \mathrm{M}$ and $2 \mu \mathrm{M}$ OF- 1 for $0,1,2$, and 3 days measured by qPCR. Data were normalized to day 0 of RANKL treatment. (D) Western blot analysis of NFATC1 protein level in murine BMCs during differentiation with $10 \mathrm{ng} / \mathrm{mL}$ RANKL and treatment with $1 \mu \mathrm{M}$ and $2 \mu \mathrm{M}$ OF-1 for $0,1,2$, and 3 days. LaminB1 was used as a loading control.

TRAP, the late-phase osteoclast marker cathepsin K (CATK), the proton generator carbonic anhydrase II (CA2), the key transcription factor NFATC1, and OC-STAMP, an essential gene involved in cell-cell fusion (Figure 5C). Suppression of gene expression by OF-1 was particularly strong at day 2 after RANKL-induced differentiation. Interestingly, the dominant negative form BRPF1A exhibited significantly decreased RNA expression levels, whereas the active acetyl-lysine binding isoform BRPF1B was not affected (Supporting Information Figure 4B). Additionally, BRPF bromodomain inhibitors had no effect on the proliferation of murine monocytic RAW246.7 cells and primary bone marrow cells, suggesting that the developed inhibitors are not cytotoxic (Supporting Information Figure 4). At the protein level, we also observed strong down-regulation of NFATC1 (Figure 5D). To further investigate other osteoclastspecific effects of the BRPF inhibitors on gene transcription, we profiled genome-wide expression with Illumina MouseWG-6 v2.0 Expression BeadChips at different time points. Significant differences $(\alpha=0.05)$ in gene expression were observed at 48 and $72 \mathrm{~h}$ but not at $24 \mathrm{~h}$ of treatment (Figure 6). Pathway analysis (reactome.org) of the top 25 genes showed that differentially expressed genes were linked either to osteoclast differentiation or 


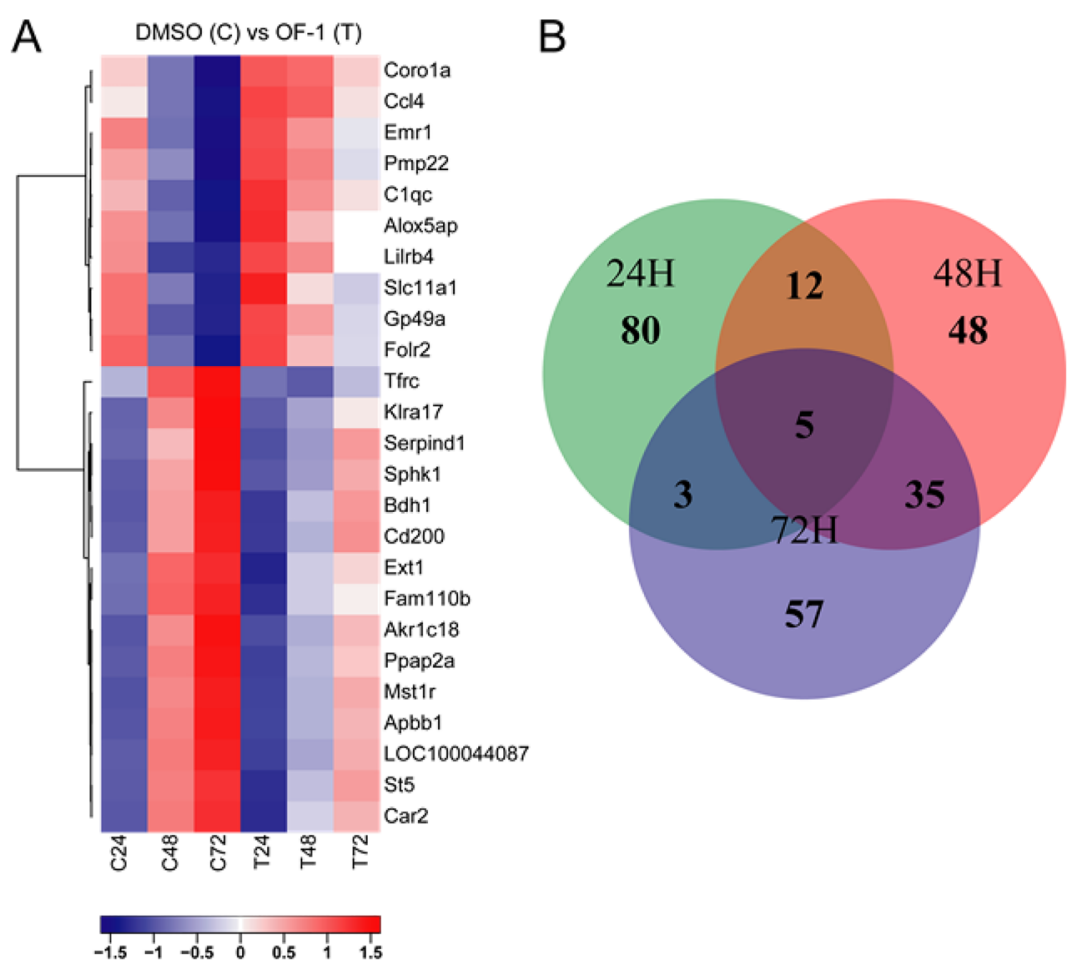

Figure 6. Microarray analysis of the effect of OF-1 on gene expression. (A) Heat map of the top 25 significant differentially expressed genes between DMSO and OF-1-treated BMCs based on sorted average $p$-value for the time points 48 and $72 \mathrm{~h}$. Red indicates higher; blue indicates lower expression. See also, Supporting Information Figures 3 and 4. (B) Venn diagram showing overlap of 100 significantly expressed genes for each time point determined by paired analysis ( $\mathrm{BH}$ multiple testing, $p$-value alpha adjustment) between DMSO and OF-1-treated $(2 \mu \mathrm{M})$ murine $\mathrm{BMCs}$ during $10 \mathrm{ng} / \mathrm{mL}$ RANKL-induced differentiation of 24,48 , or $72 \mathrm{~h}$.

to lipid metabolism, thus two pathways relevant to osteoclast differentiation and function. For example, RANKL-induced expression of Ppap2a and SphK1 which play central roles in sphingolipid de novo biosynthesis, were significantly downregulated by OF-1. These data suggest that the fusion process from macrophages to bone-resorbing "osteoclast-like" cells is interrupted by bromodomain inhibition as well.

To ensure that the inhibitors also have activity on human cells, we used human peripheral blood from healthy donors. Consistent with the results in murine cells, we observed dosedependent suppression of osteoclast differentiation in the presence of all three inhibitors and RANKL (Figure 7A). OF-1 was the only inhibitor to completely suppress the fusion into multinucleated "osteoclast-like" cells. This suggests that during osteoclastogenesis, other BRPF family members may, at least in part, functionally replace BRPF1B, which was the only BRPF family member inhibited by PFI-4 at the concentrations tested (Figure 7B). To study this process in more detail, we performed fluorescence microscopy analyses for beta-actin, VNR (vitronectin receptor; $\alpha_{\mathrm{v}} \beta_{3}$ integrin), and DNA (DAPI), to visualize the cytoskeleton with the osteoclast specific actin rings that assemble podosomes as well as nuclei. The actin ring is a hallmark of osteoclasts which forms a seal with the bone surface to create a protected compartment for bone resorption by $\mathrm{HCl}$ and proteases. Treatment with all three inhibitors led to striking decreases of F-actin rings. We also analyzed bone-resorbing activity, first by seeding the cells onto a bone mineral substrate (hydroxyapatite, HA; Osteosurface assay, Corning). Both OF-1 and NI-57 led to a significant reduction in pit formation (Figure 7C), consistent with observed decreases in differentiation. Second, cells were seeded on dentine slices, a more biologically complete substrate. As with the HA plates, resorption of dentine was also markedly reduced, including a complete block of resorption by $1 \mu \mathrm{M}$ OF-1 (Figure 7D). Consistent with this loss of activity, osteoclast marker genes CA2, CATK, NFATC1, and ACP5 (TRAP) were also down-regulated using all three BRPF inhibitors (Figure 7E and F). Matrix metallopeptidase 9 (MMP9, type IV collagenase) is a key protease secreted by osteoclasts for matrix degradation. To determine whether BRPF bromodomain inhibition leads to down regulation of MMP9, we used an ELISA to determine the protein levels in the supernatant of primary osteoclasts before and after exposure to PFI-4, OF-1, and NI-57. We found that all three inhibitors led to significant reductions in MMP9 secretion (Figure 7G). Apart from its role in normal osteoclast function, the suppression of MMP9 secretion by the studied BRPF bromodomain inhibitors may also have important implications for targeting cancer metastasis infiltration into bone, since MMP9 secretion is markedly upregulated during progression toward invasive tumors due to its central role in stromal remodelling. ${ }^{37}$

The developed three chemical probes for the bromodomain of $\mathrm{BRPF}$ led to the identification of the role of this protein interaction domain in regulating osteoclastogenesis, suggesting a key role of protein acetylation in regulating this process. Previously, Lamoureux et al. demonstrated that panBET inhibition also attenuates osteoclast differentiation. ${ }^{38}$ However, in contrast to BET inhibitors, the inhibition of BRPF bromodomains did not result in antiproliferative effects or cytotoxicity. Inhibition of BRPF bromodomains may therefore be applicable for the prevention of bone loss, and the developed chemical probes will provide an excellent chemical starting point for translational studies. BRPF is widely expressed in a variety of tissue types, and the developed probes will help to elucidate further functions of these interesting epigenetic modulators. 


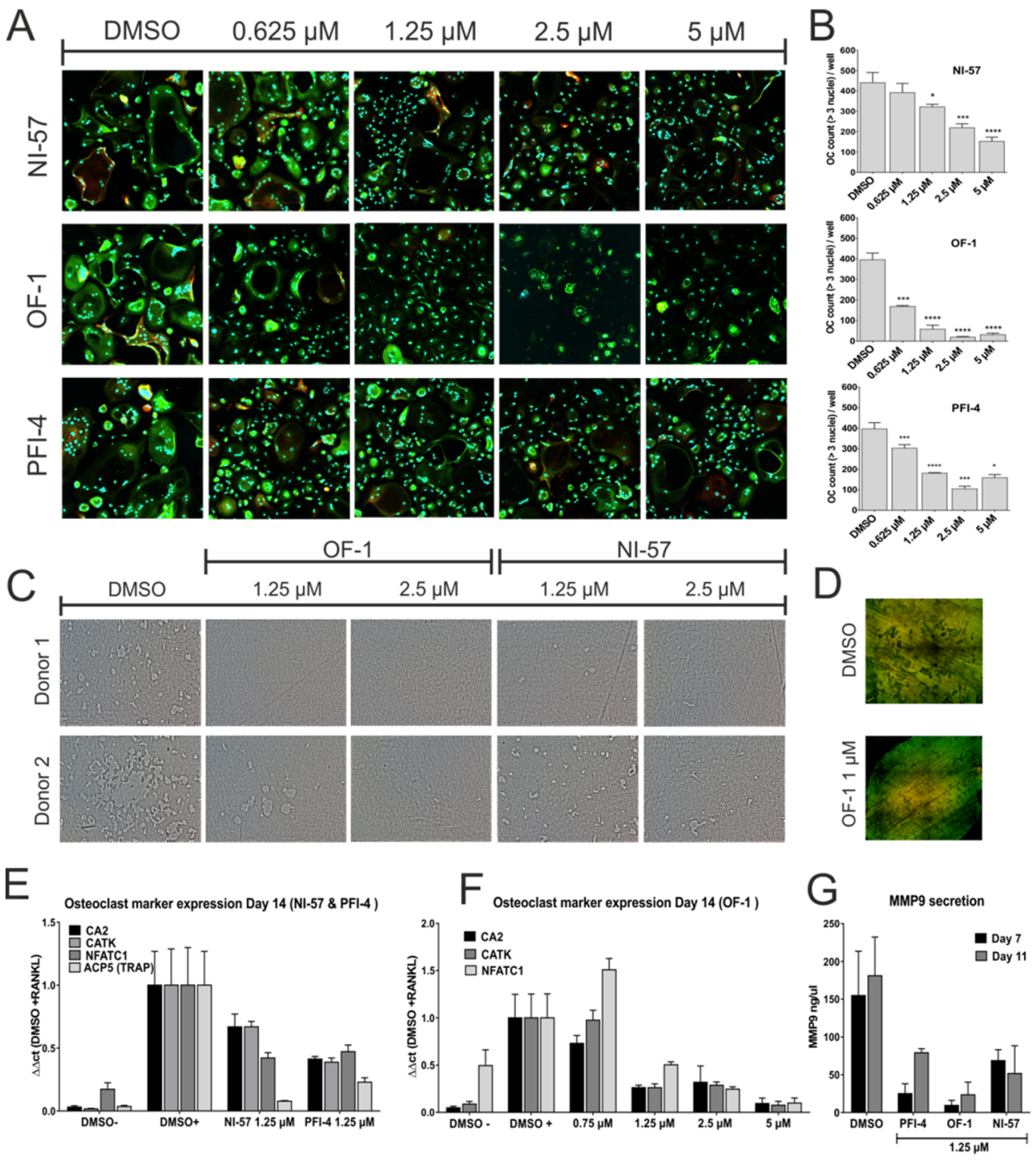

Figure 7. Inhibition of human osteoclast differentiation by panBRPF bromodomain inhibition. (A) Immunofluorescence of F-actin and VNR in primary human PBMCs differentiated into multinucleated osteoclasts by RANKL for 14 days. Osteoclasts were treated with the indicated doses of NI-57, OF-1, or PFI-4 (chemical probe set). Experiments were conducted $>10$ times with $>2$ donors each. (B) Counts of TRAP-positive multinucleated osteoclasts $(n>3)$ derived from human PBMCs after 14 days of culture with RANKL, with or without indicated doses of BRPF inhibitors. Tukey multiple comparison test $* p>0.05, * * p>0.01, * * * p>0.001, * * * p>0.0001$. (C) OF- 1 and NI-57 inhibited osteosurface degradation by human osteoclast-like cells, doses indicated. (D) OF-1 $(1 \mu \mathrm{M})$ inhibited pit formation by human osteoclast-like cells on ivory discs. (E) RNA expression of osteoclast markers by human osteoclast-like cells following 14 days of RANKL (+) and DMSO, NI-57, or PFI-4 treatment at $1.25 \mu \mathrm{M}$ or (F) OF-1 in a dose-dependent manner. (G) MMP9 concentration in the supernatant of human RANKL-induced osteoclasts treated with either PFI-4, OF-1, or NI-57 at a concentration of $1.25 \mu \mathrm{M}$ for 7 days or 11 days, respectively. See also, Supporting Information Figure 5.

\section{METHODS}

Protein Expression. All recombinant bromodomains were expressed as described in Filippakopoulos et al. ${ }^{35}$

BioLayer Interferometry (BLI). BLI experiments to determine binding kinetics were done using the Octet RED384 system by fortéBIO using bromodomains of two splice isoforms of BRPF1A and BRPF1B that were biotinylated during recombinant expression using a BirA (Biotin transferase) overexpressing bacterial host (BL21 DE3) in conjunction with a C-terminal AVI-tag. Biotinylated proteins were immobilized on super streptavidin biosensors, which were subsequently quenched with L-biotin. Data analysis software provided by fortéBIO was used to calculate binding constants from the interference data.

AlphaScreen. Assays were performed as described previously with minor modifications. ${ }^{39}$ Plates filled with $5 \mu \mathrm{L}$ of the assay buffer followed by $7 \mu \mathrm{L}$ of biotinylated peptide [H-YSGRGKacGGKacGLGKacGGA KacRHRK(Biotin)-OH for BRD1, BRD4, BRPF1B, and BRPF3 or
YQTARKSTGGK(ac)APRKQLATKAK(biotin)-OH for TIF1 $\alpha$ ] and His-tagged protein to achieve final assay concentrations of $25-100 \mathrm{nM}$ depending on the dose-response curve for each individual protein.

Murine and Human Osteoclast Differentiation. Primary mouse bone marrow mononuclear cells (BMMC) were obtained, differentiated, and stained for TRAP as previously described. ${ }^{40}$ Briefly, marrow cell suspensions from PBS flushed bones (two tibiae, two femora) from 2 to 3 week old mice were centrifuged at $1000 \mathrm{~g}$ for $5 \mathrm{~min}$ and plated in $\alpha \mathrm{MEM} / 10 \%$ FBS with low MCSF $(10 \mathrm{ng} / \mathrm{mL}$, human recombinant MCSF; Chiron) and incubated at $37^{\circ} \mathrm{C}$ and $5 \% \mathrm{CO}_{2}$. After 3 days, fresh medium containing high MCSF $(75 \mathrm{ng} / \mathrm{mL}$ ) was added for 2 more days, and then the medium was supplemented with RANKL (R\&D Systems, Minneapolis) at $1 \mathrm{ng} / \mathrm{mL}$ unless otherwise noted, for the times indicated. Differentiation into osteoclasts was scored by counting multinucleated (three or more nuclei) TRAP-positive cells. Primary human peripheral blood (Oxford NHS Blood bank, NCI0622) mononuclear cells (PBMCs) were collected from a Histopaque generated buffy coat after gradient 
centrifugation at $20 \mathrm{~min}$ and $500 \mathrm{~g}$, brakes off. The $\mathrm{CD} 14^{+}$monocyte fraction was obtained by on-column $\mathrm{CD} 14^{+}$-MACS bead isolation (Miltenyi Biotech, Surrey, UK), washed twice with MACS buffer, and seeded at a density of $50000 \mathrm{c} / \mathrm{mL}$ in $\alpha \mathrm{MEM} / 10 \% \mathrm{FCS}$ supplemented with $25 \mathrm{ng} / \mathrm{mL}$ MCSF (Peprotech). After 6 days at $37^{\circ} \mathrm{C}, 5 \% \mathrm{CO}_{2}$ treatment with either OF-1, NI-57, or PFI-4 with and without $50 \mathrm{ng} / \mathrm{mL}$ RANKL (Peprotech) was started. Media were changed with fresh compounds every 3-4 days. After 14-21 days, cells were fixed and stained for TRAP or lysed in RLT/2-mercaptoethanol RNA lysis buffer for RNA isolation.

Immunofluorescence. Fixed osteoclast-like cells were permabilized in $0.5 \%$ Triton X-100 at RT (RT) for $20 \mathrm{~min}$. Blocking of nonspecific binding was carried out with $3 \%$ FBS/PBS for at least $1 \mathrm{~h}$ at RT. Primary antibodies for VNR and F-actin were incubated overnight in 3\%FCS/ PBS (1:500). After washes with PBS, secondary fluorescence coupled antimouse and antirabbit antibody were applied for $1 \mathrm{~h}$ in the dark, at RT. DAPI/PBS was added for $5 \mathrm{~min}$, and fluorescence was measured by confocal microscopy (Zeiss).

Bone Resorption Assays. PBMCs were isolated and seeded onto either osteosurface assay plates (BD biosciences) or self-cut dentine slices from ivory (provided by Edward Hookway, Botnar institute). After 14 days of differentiation, cells were removed from osteosurface or dentine slices. The amount of osteoclast-mediated pits in the osteosurface was assessed by phase contrast. Dentine pits were imaged with confocal microscopy.

Western Blot and ELISA. Murine osteoclast-like cells were PBS washed, homogenized and lysed in RIPA buffer $(50 \mathrm{mM}$ Tris- $\mathrm{HCl}$, $\mathrm{pH} 7.4 ; 150 \mathrm{mM} \mathrm{NaCl} ; 1 \% \mathrm{NP}-40 ; 0.5 \% \mathrm{Na}$-deoxycholate; $0.1 \% \mathrm{SDS}$; $2 \mathrm{mM}$ EDTA; $10 \mathrm{mM} \mathrm{NaF}$ ) containing protease inhibitors (Pierce, according to manufacturer's specifications) with a $1 \mathrm{~mL}$ syringe and a 23-gauge needle. After centrifugation at $15000 \mathrm{rpm}$ at $4{ }^{\circ} \mathrm{C}$ for $10 \mathrm{~min}$, supernatants were frozen at $-80{ }^{\circ} \mathrm{C}$. Protein concentrations were assayed using a Pierce BCA Kit, and $30 \mu \mathrm{g}$ of protein per lane was subjected on $10 \%$ SDS gel. Proteins were electroblotted onto PVDF, and blots were probed overnight at $4{ }^{\circ} \mathrm{C}$ (anti-NFATc1 (1:500; 7A6: sc-7294 mouse monoclonal, Santa Cruz) or anti-Lamin B1 (1:10 000; ab133741 rabbit monoclonal, Abcam). Secondary antibodies were HRP-conjugated sheep antimouse (1:5000 for Nfatc1; \#NA93IV; GE Healthcare, Piscataway, NJ) or HRP-conjugated goat antirabbit (1:5000 for Lamin B1; \# P0448; Dako, Carpinteria, CA). MMP9 secretion in the human osteoclast supernatant was determined by a MMP9 ELISA (R\&D, DMP900) according to the manufacturer's instructions. The supernatant $(50 \mu \mathrm{L} /$ well $)$ was collected, frozen at $-20^{\circ} \mathrm{C}$, and 100 -fold diluted for the assay. Absorbance was measured at $440 \mathrm{~nm}$.

Microarray and Bioinformatics Analysis. Murine bone marrow stem cells were differentiated into osteoclast-like cells as described above. Cells were PBS-washed and lysed in RLT-Buffer (including 2-Mercaptoethanol; Qiagen, UK), and RNA was isolated via RNeasy Plus Mini Kit (Quiagen, UK). After confirmation of RNA quality (RIN = $2 \pm 0.1$ ), cDNA synthesis of approximately $1000 \mathrm{ng}$ of RNA with a High Capacity cDNA Reverse Transcription Kit (Applied Biosystems, UK) was carried out. A total of $500 \mathrm{ng} / \mu \mathrm{L}$ cDNA was subjected to MouseWG-6 v2.0 Expression BeadChips (Illumina) at the Department of Pathology, University of Cambridge. Microarray data are available in the ArrayExpress database (www.ebi.ac.uk/arrayexpress) under accession number E-MTAB-4155. Gene expression data underwent log2 transformation and quantile normalization. Genes were filtered based on their variation across the experiments, i.e., excluding genes with a standard deviation of expression lower than 0.1. For genes with multiple probes, only the highest variable probe was selected for further analysis. Differently expressed genes were selected using LIMMA (Linear Models for Microarray Data) in a paired analysis for the replicates. This method resulted in p-values adjusted for multiple testing using the BenjaminiHochberg procedure, and $\alpha=0.01$ was used to define significance. Microarray analyses were performed in R (v3.0.1) using the packages Limma (3.16.8) for differential expression and Venn diagram (1.6.7), plotrix (3.5-7), gtools (3.4.1), and gplots (2.14.1) for visualization. Pathway analysis of the top 25 differentially expressed genes with a Jaccard distance coefficient of $p>0.05$ (Reactome.org) was performed. The REACT_111217 pathway (metabolism of lipids and lipoproteins) with at least more than four enriched genes was set as the most significant (entities p-value 0.045). Additional methods' descriptions are available in the Supporting Information.

\section{ASSOCIATED CONTENT}

\section{Supporting Information}

The Supporting Information is available free of charge on the ACS Publications website at DOI: 10.1021/acschembio.7b00481.

Additional experimental data methods and figures (PDF)

\section{AUTHOR INFORMATION}

\section{Corresponding Authors}

*E-mail: mark.birnbaum@merrimack.edu.

*E-mail: knapp@pharmchem.uni-frankfurt.de.

ORCID $\odot$

Paul V. Fish: 0000-0002-2117-2173

Paul E. Brennan: 0000-0002-8950-7646

Stefan Knapp: 0000-0001-5995-6494

\section{Author Contributions}

J.C.M., C.T., O.F., H.W., S.-Y.H., R.G.v.S., J.-P.L., C.R., D.H., C.Y., B.S.G., V.F., P.S., D.L.D., N.M.I., and E.D.B. conducted the experiments. D.R.O., P.V.F., B.H., U.C.T.O., F.B., P.E.B., S.M., A.C.G., P.R.O., M.J.B., an S.K. designed the experiments and supervised research. M.J.B. and S.K. wrote the paper. All authors read and approved the final version of the manuscript.

\section{Notes}

The authors declare no competing financial interest.

\section{ACKNOWLEDGMENTS}

J.-P.L. was supported by a postdoctoral fellowship from CIHR and by a TD Bank Health Research Fellowship at the LunenfeldTanenbaum Research Institute. Work in the Gingras lab was supported by the Canadian Institutes of Health Research (Foundation grant FDN143301). A.C.G. is the Canada Research Chair in Functional Proteomics and the Lea Reichmann Chair in Cancer Proteomics. U.C.T.O. is supported by Arthritis Research UK (program grant 20522) and the Rosetrees Trust. J.C.M. was supported by a Bayer postdoctoral fellowship. The SGC is a registered charity (number 1097737) that receives funds from AbbVie, Bayer Pharma AG, Boehringer Ingelheim, Canada Foundation for Innovation, Eshelman Institute for Innovation, Genome Canada through Ontario Genomics Institute, Janssen, Merck \& Co., Novartis Pharma AG, Ontario Ministry of Economic Development and Innovation, Pfizer, São Paulo Research Foundation-FAPESP, Takeda, the Centre of Excellence Initiative Macromolecular Complexes (CEF) at Frankfurt University, and the Wellcome Trust. H.W., S.-Y.H., P.R.O., and M.J.B. were supported in part by NIAMS grant AR061504 and NIDCR grant DE 007444 (U.S. National Institutes of Health). M.J.B. was supported in part by Merrimack College.

\section{REFERENCES}

(1) Falkenberg, K. J., and Johnstone, R. W. (2014) Histone deacetylases and their inhibitors in cancer, neurological diseases and immune disorders. Nat. Rev. Drug Discovery 13, 673-691.

(2) Verdin, E., and Ott, M. (2014) 50 years of protein acetylation: from gene regulation to epigenetics, metabolism and beyond. Nat. Rev. Mol. Cell Biol. 16, 258-264.

(3) Filippakopoulos, P., and Knapp, S. (2012) The bromodomain interaction module. FEBS Lett. 586, 2692-2704. 
(4) Vidler, L. R., Brown, N., Knapp, S., and Hoelder, S. (2012) Druggability analysis and structural classification of bromodomain acetyl-lysine binding sites. J. Med. Chem. 55, 7346-7359.

(5) Filippakopoulos, P., Qi, J., Picaud, S., Shen, Y., Smith, W. B., Fedorov, O., Morse, E. M., Keates, T., Hickman, T. T., Felletar, I., Philpott, M., Munro, S., McKeown, M. R., Wang, Y., Christie, A. L., West, N., Cameron, M. J., Schwartz, B., Heightman, T. D., La Thangue, N., French, C. A., Wiest, O., Kung, A. L., Knapp, S., and Bradner, J. E. (2010) Selective inhibition of BET bromodomains. Nature 468, 10671073.

(6) Picaud, S., Da Costa, D., Thanasopoulou, A., Filippakopoulos, P., Fish, P. V., Philpott, M., Fedorov, O., Brennan, P., Bunnage, M. E., Owen, D. R., Bradner, J. E., Taniere, P., O'Sullivan, B., Muller, S., Schwaller, J., Stankovic, T., and Knapp, S. (2013) PFI-1, a highly selective protein interaction inhibitor, targeting BET Bromodomains. Cancer Res. 73, 3336-3346.

(7) Nicodeme, E., Jeffrey, K. L., Schaefer, U., Beinke, S., Dewell, S., Chung, C. W., Chandwani, R., Marazzi, I., Wilson, P., Coste, H., White, J., Kirilovsky, J., Rice, C. M., Lora, J. M., Prinjha, R. K., Lee, K., and Tarakhovsky, A. (2010) Suppression of inflammation by a synthetic histone mimic. Nature 468, 1119-1123.

(8) Dawson, M. A., Prinjha, R. K., Dittmann, A., Giotopoulos, G., Bantscheff, M., Chan, W. I., Robson, S. C., Chung, C. W., Hopf, C., Savitski, M. M., Huthmacher, C., Gudgin, E., Lugo, D., Beinke, S., Chapman, T. D., Roberts, E. J., Soden, P. E., Auger, K. R., Mirguet, O., Doehner, K., Delwel, R., Burnett, A. K., Jeffrey, P., Drewes, G., Lee, K., Huntly, B. J., and Kouzarides, T. (2011) Inhibition of BET recruitment to chromatin as an effective treatment for MLL-fusion leukaemia. Nature $478,529-533$.

(9) Filippakopoulos, P., and Knapp, S. (2014) Targeting bromodomains: epigenetic readers of lysine acetylation. Nat. Rev. Drug Discovery 13, 337-356.

(10) Clark, P. G., Vieira, L. C., Tallant, C., Fedorov, O., Singleton, D. C., Rogers, C. M., Monteiro, O. P., Bennett, J. M., Baronio, R., Muller, S., Daniels, D. L., Mendez, J., Knapp, S., Brennan, P. E., and Dixon, D. J. (2015) LP99: Discovery and Synthesis of the First Selective BRD7/9 Bromodomain Inhibitor. Angew. Chem., Int. Ed. 54, 6217-6221.

(11) Chen, P., Chaikuad, A., Bamborough, P., Bantscheff, M., Bountra, C., Chung, C. W., Fedorov, O., Grandi, P., Jung, D., Lesniak, R., Lindon, M., Muller, S., Philpott, M., Prinjha, R., Rogers, C., Selenski, C., Tallant, C., Werner, T., Willson, T. M., Knapp, S., and Drewry, D. H. (2016) Discovery and Characterization of GSK2801, a Selective Chemical Probe for the Bromodomains BAZ2A and BAZ2B. J. Med. Chem. 59, 1410.

(12) Drouin, L., McGrath, S., Vidler, L. R., Chaikuad, A., Monteiro, O., Tallant, C., Philpott, M., Rogers, C., Fedorov, O., Liu, M., Akhtar, W., Hayes, A., Raynaud, F., Muller, S., Knapp, S., and Hoelder, S. (2015) Structure enabled design of BAZ2-ICR, a chemical probe targeting the bromodomains of BAZ2A and BAZ2B. J. Med. Chem. 58, 2553-2559.

(13) Hay, D. A., Fedorov, O., Martin, S., Singleton, D. C., Tallant, C., Wells, C., Picaud, S., Philpott, M., Monteiro, O. P., Rogers, C. M., Conway, S. J., Rooney, T. P., Tumber, A., Yapp, C., Filippakopoulos, P., Bunnage, M. E., Muller, S., Knapp, S., Schofield, C. J., and Brennan, P. E. (2014) Discovery and optimization of small-molecule ligands for the CBP/p300 bromodomains. J. Am. Chem. Soc. 136, 9308-9319.

(14) Theodoulou, N. H., Bamborough, P., Bannister, A. J., Becher, I., Bit, R. A., Che, K. H., Chung, C. W., Dittmann, A., Drewes, G., Drewry, D. H., Gordon, L., Grandi, P., Leveridge, M., Lindon, M., Michon, A. M., Molnar, J., Robson, S. C., Tomkinson, N. C., Kouzarides, T., Prinjha, R. K., and Humphreys, P. G. (2016) Discovery of I-BRD9, a Selective Cell Active Chemical Probe for Bromodomain Containing Protein 9 Inhibition. J. Med. Chem. 59, 1425.

(15) Fedorov, O., Castex, J., Tallant, C., Owen, D. R., Martin, S., Aldeghi, M., Monteiro, O., Filippakopoulos, P., Picaud, S., Trzupek, J. D., Gerstenberger, B. S., Bountra, C., Willmann, D., Wells, C., Philpott, M., Rogers, C., Biggin, P. C., Brennan, P. E., Bunnage, M. E., Schule, R., Gunther, T., Knapp, S., and Muller, S. (2015) Selective targeting of the $\mathrm{BRG} / \mathrm{PB} 1$ bromodomains impairs embryonic and trophoblast stem cell maintenance. Sci. Adv. 1, e1500723.
(16) Picaud, S., Fedorov, O., Thanasopoulou, A., Leonards, K., Jones, K., Meier, J., Olzscha, H., Monteiro, O., Martin, S., Philpott, M., Tumber, A., Filippakopoulos, P., Yapp, C., Wells, C., Che, K. H., Bannister, A., Robson, S., Kumar, U., Parr, N., Lee, K., Lugo, D., Jeffrey, P., Taylor, S., Vecellio, M. L., Bountra, C., Brennan, P. E., O’Mahony, A., Velichko, S., Muller, S., Hay, D., Daniels, D. L., Urh, M., La Thangue, N. B., Kouzarides, T., Prinjha, R., Schwaller, J., and Knapp, S. (2015) Generation of a Selective Small Molecule Inhibitor of the CBP/p300 Bromodomain for Leukemia Therapy. Cancer Res. 75, 5106-5119.

(17) Martin, L. J., Koegl, M., Bader, G., Cockcroft, X. L., Fedorov, O., Fiegen, D., Gerstberger, T., Hofmann, M. H., Hohmann, A. F., Kessler, D., Knapp, S., Knesl, P., Kornigg, S., Muller, S., Nar, H., Rogers, C., Rumpel, K., Schaaf, O., Steurer, S., Tallant, C., Vakoc, C. R., Zeeb, M., Zoephel, A., Pearson, M., Boehmelt, G., and McConnell, D. (2016) Structure-Based Design of an in Vivo Active Selective BRD9 Inhibitor. J. Med. Chem. 59, 4462-4475.

(18) Popp, T. A., Tallant, C., Rogers, C., Fedorov, O., Brennan, P. E., Muller, S., Knapp, S., and Bracher, F. (2016) Development of Selective CBP/P300 Benzoxazepine Bromodomain Inhibitors. J. Med. Chem. 59, 8889-8912.

(19) Demont, E. H., Bamborough, P., Chung, C. W., Craggs, P. D., Fallon, D., Gordon, L. J., Grandi, P., Hobbs, C. I., Hussain, J., Jones, E. J., Le Gall, A., Michon, A. M., Mitchell, D. J., Prinjha, R. K., Roberts, A. D., Sheppard, R. J., and Watson, R. J. (2014) 1,3-Dimethyl Benzimidazolones Are Potent, Selective Inhibitors of the BRPF1 Bromodomain. ACS Med. Chem. Lett. 5, 1190-1195.

(20) Palmer, W. S., Poncet-Montange, G., Liu, G., Petrocchi, A., Reyna, N., Subramanian, G., Theroff, J., Yau, A., Kost-Alimova, M., Bardenhagen, J. P., Leo, E., Shepard, H. E., Tieu, T. N., Shi, X., Zhan, Y., Zhao, S., Barton, M. C., Draetta, G., Toniatti, C., Jones, P., Geck Do, M., and Andersen, J. N. (2016) Structure-Guided Design of IACS-9571, a Selective High-Affinity Dual TRIM24-BRPF1 Bromodomain Inhibitor. J. Med. Chem. 59, 1440.

(21) Bennett, J., Fedorov, O., Tallant, C., Monteiro, O., Meier, J., Gamble, V., Savitsky, P., Nunez-Alonso, G. A., Haendler, B., Rogers, C., Brennan, P. E., Muller, S., and Knapp, S. (2016) Discovery of a Chemical Tool Inhibitor Targeting the Bromodomains of TRIM24 and BRPF. J. Med. Chem. 59, 1642.

(22) Vezzoli, A., Bonadies, N., Allen, M. D., Freund, S. M., Santiveri, C. M., Kvinlaug, B. T., Huntly, B. J., Gottgens, B., and Bycroft, M. (2010) Molecular basis of histone H3K36me3 recognition by the PWWP domain of Brpf1. Nat. Struct. Mol. Biol. 17, 617-619.

(23) Hibiya, K., Katsumoto, T., Kondo, T., Kitabayashi, I., and Kudo, A. (2009) Brpf1, a subunit of the MOZ. histone acetyl transferase complex, maintains expression of anterior and posterior Hox genes for proper patterning of craniofacial and caudal skeletons. Dev. Biol. 329, 176-190.

(24) Lalonde, M. E., Avvakumov, N., Glass, K. C., Joncas, F. H., Saksouk, N., Holliday, M., Paquet, E., Yan, K., Tong, Q., Klein, B. J., Tan, S., Yang, X. J., Kutateladze, T. G., and Cote, J. (2013) Exchange of associated factors directs a switch in $\mathrm{HBO} 1$ acetyltransferase histone tail specificity. Genes Dev. 27, 2009-2024.

(25) Lubula, M. Y., Eckenroth, B. E., Carlson, S., Poplawski, A., Chruszcz, M., and Glass, K. C. (2014) Structural insights into recognition of acetylated histone ligands by the BRPF1 bromodomain. FEBS Lett. 588, 3844-3854.

(26) Laue, K., Daujat, S., Crump, J. G., Plaster, N., Roehl, H. H., Kimmel, C. B., Schneider, R, and Hammerschmidt, M. (2008) The multidomain protein Brpf1 binds histones and is required for Hox gene expression and segmental identity. Development 135, 1935-1946.

(27) Katsumoto, T., Aikawa, Y., Iwama, A., Ueda, S., Ichikawa, H., Ochiya, T., and Kitabayashi, I. (2006) MOZ is essential for maintenance of hematopoietic stem cells. Genes Dev. 20, 1321-1330.

(28) Ullah, M., Pelletier, N., Xiao, L., Zhao, S. P., Wang, K., Degerny, C., Tahmasebi, S., Cayrou, C., Doyon, Y., Goh, S. L., Champagne, N., Cote, J., and Yang, X. J. (2008) Molecular architecture of quartet MOZ/ MORF histone acetyltransferase complexes. Mol. Cell. Biol. 28, 68286843.

(29) Mishima, Y., Miyagi, S., Saraya, A., Negishi, M., Endoh, M., Endo, T. A., Toyoda, T., Shinga, J., Katsumoto, T., Chiba, T., Yamaguchi, N., 
Kitabayashi, I., Koseki, H., and Iwama, A. (2011) The Hbo1-Brd1/Brpf2 complex is responsible for global acetylation of $\mathrm{H} 3 \mathrm{~K} 14$ and required for fetal liver erythropoiesis. Blood 118, 2443-2453.

(30) Nyegaard, M., Severinsen, J. E., Als, T. D., Hedemand, A., Straarup, S., Nordentoft, M., McQuillin, A., Bass, N., Lawrence, J., Thirumalai, S., Pereira, A. C., Kandaswamy, R., Lydall, G. J., Sklar, P., Scolnick, E., Purcell, S., Curtis, D., Gurling, H. M., Mortensen, P. B., Mors, O., and Borglum, A. D. (2010) Support of association between BRD1 and both schizophrenia and bipolar affective disorder. Am. J. Med. Genet., Part B 153B, 582-591.

(31) Igoe, E., Fedorov, O., Tallant, C., Savitsky, P., Rogers, C., Owen, D., Deb, G., Somervaille, T., Andrews, D., Jones, N., Cheasty, A., Ryder, H., Brennan, P., Müller, S., Knapp, S., Fish, P., and Bayle, E. D. (2017) Design of a biased potent small molecule inhibitor of the bromodomain and PHD finger-containing (BRPF) proteins suitable for cellular and in vivo studies (2017). J. Med. Chem. 60, 668-680.

(32) Igoe, N., Bayle, E. D., Tallant, C., Fedorov, O., Meier, J. C., Savitsky, P., Rogers, C., Morias, Y., Scholze, S., Boyd, H., Cunoosamy, D., Andrews, D. M., Cheasty, A., Brennan, P. E., Muller, S., Knapp, S., and Fish, P. V. (2017) Design of a chemical probe for the Bromodomain and Plant Homeodomain Finger-containing (BRPF) family of proteins (2017). J. Med. Chem. 60, 6998-7011.

(33) Philpott, M., Rogers, C. M., Yapp, C., Wells, C., Lambert, J. P., Strain-Damerell, C., Burgess-Brown, N. A., Gingras, A. C., Knapp, S., and Muller, S. (2014) Assessing cellular efficacy of bromodomain inhibitors using fluorescence recovery after photobleaching. Epigenet. Chromatin 7, 14.

(34) Tallant, C., Valentini, E., Fedorov, O., Overvoorde, L., Ferguson, F. M., Filippakopoulos, P., Svergun, D. I., Knapp, S., and Ciulli, A. (2015) Molecular basis of histone tail recognition by human TIP5 PHD finger and bromodomain of the chromatin remodeling complex NoRC. Structure 23, 80-92.

(35) Filippakopoulos, P., Picaud, S., Mangos, M., Keates, T., Lambert, J. P., Barsyte-Lovejoy, D., Felletar, I., Volkmer, R, Muller, S., Pawson, T., Gingras, A. C., Arrowsmith, C. H., and Knapp, S. (2012) Histone recognition and large-scale structural analysis of the human bromodomain family. Cell 149, 214-231.

(36) Roux, K. J., Kim, D. I., and Burke, B. (2013) BioID: a screen for protein-protein interactions. Curr. Protoc Protein Sci. 74 (19), 23.

(37) Kitamura, T., Qian, B. Z., and Pollard, J. W. (2015) Immune cell promotion of metastasis. Nat. Rev. Immunol. 15, 73-86.

(38) Lamoureux, F., Baud'huin, M., Rodriguez Calleja, L., Jacques, C., Berreur, M., Redini, F., Lecanda, F., Bradner, J. E., Heymann, D., and Ory, B. (2014) Selective inhibition of BET bromodomain epigenetic signalling interferes with the bone-associated tumour vicious cycle. Nat. Commun. 5, 3511.

(39) Philpott, M., Yang, J., Tumber, T., Fedorov, O., Uttarkar, S., Filippakopoulos, P., Picaud, S., Keates, T., Felletar, I., Ciulli, A., Knapp, S., and Heightman, T. D. (2011) Bromodomain-peptide displacement assays for interactome mapping and inhibitor discovery. Mol. BioSyst. 7, 2899-2908.

(40) Yang, M., Birnbaum, M. J., MacKay, C. A., Mason-Savas, A., Thompson, B., and Odgren, P. R. (2008) Osteoclast stimulatory transmembrane protein (OC-STAMP), a novel protein induced by RANKL that promotes osteoclast differentiation. J. Cell. Physiol. 215, 497-505. 